\title{
$\begin{array}{llllllllll}S & T & O & C & H & A & S & T & I & C\end{array}$ \\ D I F F E R E N T I A L
}

EQUATIONS IN SCIENCE

A N D ENG I NEER I N G 
This page is intentionally left blank 


\section{Douglas Henderson}

Brigham Young University, USA

\section{Peter Plaschko}

Universidad Autónoma Metropolitana, Mexico 


\section{Published by}

World Scientific Publishing Co. Pte. Ltd.

5 Toh Tuck Link, Singapore 596224

USA office: 27 Warren Street, Suite 401-402, Hackensack, NJ 07601

UK office: 57 Shelton Street, Covent Garden, London WC2H 9HE

\section{British Library Cataloguing-in-Publication Data}

A catalogue record for this book is available from the British Library.

\section{STOCHASTIC DIFFERENTIAL EQUATIONS IN SCIENCE AND ENGINEERING (With CD-ROM)}

Copyright $\odot 2006$ by World Scientific Publishing Co. Pte. Ltd.

All rights reserved. This book, or parts thereof, may not be reproduced in any form or by any means, electronic or mechanical, including photocopying, recording or any information storage and retrieval system now known or to be invented, without written permission from the Publisher.

For photocopying of material in this volume, please pay a copying fee through the Copyright Clearance Center, Inc., 222 Rosewood Drive, Danvers, MA 01923, USA. In this case permission to photocopy is not required from the publisher.

ISBN $981-256-296-6$ 
To Rose-Marie Henderson

A good friend and spouse 
This page is intentionally left blank 


\section{PREFACE}

This book arose from a friendship formed when we were both faculty members of the Department of Physics, Universidad Autonoma Metropolitana, Iztapalapa Campus, in Mexico City. Plaschko was teaching an intermediate to advanced course in mathematical physics. He had written, with Klaus Brod, a book entitled, "Hoehere Mathematische Methoden fuer Ingenieure und Physiker", that Henderson admired and suggested that be translated into English and be updated and perhaps expanded somewhat.

However, we both prefer new projects and this suggested instead that a book on Stochastic Differential Equations be written and this project was born. This is an important emerging field. From its inception with Newton, physical science was dominated by the idea of determinism. Everything was thought to be determined by a set of second order differential equations, Newton's equations, from which everything could be determined, at least in principle, if the initial conditions were known. To be sure, an actual analytic solution would not be possible for a complex system since the number of dynamical equations would be enormous; even so, determinism prevailed. This idea took hold even to the point that some philosophers began to speculate that humans had no free will; our lives were determined entirely by some set of initial conditions. In this view, even before the authors started to write, the contents of this book were determined by a set of initial conditions in the distant past. Dogmatic Marxism endorsed such ideas, although perhaps not so extremely.

Deterministic Newtonian mechanics yielded brilliant successes. Most astronomical events could be predicted with great accuracy. 
Even in case of a few difficulties, such as the orbit of Mercury, Newtonian mechanics could be replaced satisfactorily by equally deterministric general relativity. A little more than a century ago, the case for determinism was challenged. The seemingly random motion of the Brownian motion of suspended particles was observed as was the sudden transition of the flow of a fluid past an object or obstacle from lamanar flow to chaotic turbulence. Recent studies have shown that some seemingly chaotic motion is not necessarily inconsistent with determinism (we can call this quasi-chaos). Even so, such problems are best studied using probablistic notions. Quantum theory has shown that the motion of particles at the atomic level is fundamentally nondeterministic. Heisenberg showed that there were limits to the precision with which physical properties could be determined. One can only assign a probablity for the value of a physical quantity. The consequence of this idea can be manifest even on a macroscopic scale. The third law of thermodynamics is an example.

Stochastic differential equations, the subject of this monograph, is an interesting extension of the deterministic differential equations that can be applied to Brownian motion as well as other problems. It arose from the work of Einstein and Smoluchowski among others. Recent years have seen rapid advances due to the development of the calculii of Ito and Stratonovich.

We were both trained as mathematicians and scientists and our goal is to present the ideas of stochastic differential equations in a short monograph in a manner that is useful for scientists and engineers, rather than mathematicians and without overpowering mathematical rigor. We presume that the reader has some, but not extensive, knowledge of probability theory. Chapter 1 provides a reminder and introduction to and definition of some fundamental ideas and quantities, including the ideas of Ito and Stratonovich. Stochastic differential equations and the Fokker-Planck equation are presented in Chapters 2 and 3. More advanced applications follow in Chapter 4 . The book concludes with a presentation of some numerical routines for the solution of ordinary stochastic differential equations. Each chapter contains a set of exercises whose purpose is to aid the reader in understanding the material. A CD-ROM that provides 
MATHEMATICA and FORTRAN programs to assist the reader with the exercises, numerical routines and generating figures accompanies the text.

Douglas Henderson

Peter Plaschko

Provo Utah, USA

Mexico City DF, Mexico

June, 2006 
This page is intentionally left blank 


\section{CONTENTS}

Preface vii

Introduction $\quad \mathrm{xV}$

Glossary $\quad$ xxi

1. Stochastic Variables and Stochastic Processes 1

1.1. Probability Theory 1

1.2. Averages 4

1.3. Stochastic Processes, the Kolmogorov Criterion and Martingales 9

1.4. The Gaussian Distribution and Limit Theorems 14

1.4.1. The central limit theorem 16

1.4.2. The law of the iterated logarithm 17

1.5. Transformation of Stochastic Variables 17

1.6. The Markov Property 19

1.6.1. Stationary Markov processes 20

1.7. The Brownian Motion 21

1.8. Stochastic Integrals 28

1.9. The Ito Formula 38

1.9. The Ito Formula 38

Appendix $\quad 45$

$\begin{array}{ll}\text { Exercises } & 49\end{array}$

2. Stochastic Differential Equations 55

2.1. One-Dimensional Equations 56

2.1.1. Growth of populations $\quad 56$

2.1.2. Stratonovich equations 58 
2.1.3. The problem of Ornstein-Uhlenbeck and the Maxwell distribution

2.1.4. The reduction method 63

2.1.5. Verification of solutions $\quad 65$

2.2. White and Colored Noise, Spectra 67

2.3. The Stochastic Pendulum 70

2.3.1. Stochastic excitation 72

2.3.2. Stochastic damping $(\beta=\gamma=0 ; \alpha \neq 0)$

2.4. The General Linear SDE $\quad 76$

2.5. A Class of Nonlinear SDE $\quad 79$

2.6. Existence and Uniqueness of Solutions 84

$\begin{array}{ll}\text { Exercises } & 87\end{array}$

3. The Fokker-Planck Equation 91

3.1. The Master Equation 91

3.2. The Derivation of the Fokker-Planck Equation 95

3.3. The Relation Between the Fokker-Planck Equation and Ordinary SDE's 98

3.4. Solutions to the Fokker-Planck Equation 104

3.5. Lyapunov Exponents and Stability 107

3.6. Stochastic Bifurcations 110

3.6.1. First order SDE's 110

3.6.2. Higher order SDE's 112

Appendix A. Small Noise Intensities and the Influence

of Randomness Limit Cycles 117

Appendix B.1 The method of Lyapunov functions 124

Appendix B.2 The method of linearization 128

$\begin{array}{ll}\text { Exercises } & 130\end{array}$

4. Advanced Topics 135

4.1. Stochastic Partial Differential Equations 135

4.2. Stochastic Boundary and Initial Conditions 141

4.2.1. A deterministic one-dimensional wave equation 141

4.2.2. Stochastic initial conditions 144

4.3. Stochastic Eigenvalue Equations 147

$\begin{array}{ll}\text { 4.3.1. Introduction } & 147\end{array}$

4.3.2. Mathematical methods 148 
4.3.3. Examples of exactly soluble problems $\quad 152$

4.3.4. Probability laws and moments of the eigenvalues 156

4.4. Stochastic Economics 160

4.4.1. Introduction 160

4.4.2. The Black-Scholes market 162

Exercises 164

5. Numerical Solutions of Ordinary Stochastic Differential Equations 167

5.1. Random Numbers Generators and Applications $\quad 167$

5.1.1. Testing of random numbers 168

5.2. The Convergence of Stochastic Sequences 173

5.3. The Monte Carlo Integration 175

5.4. The Brownian Motion and Simple Algorithms for SDE's 179

5.5. The Ito-Taylor Expansion of the Solution of a 1D SDE 181

5.6. Modified 1D Milstein Schemes 187

5.7. The Ito-Taylor Expansion for N-dimensional SDE's 189

5.8. Higher Order Approximations 193

5.9. Strong and Weak Approximations and the Order of the Approximation 196

Exercises 201

$\begin{array}{ll}\text { References } & 205\end{array}$

$\begin{array}{ll}\text { Fortran Programs } & 211\end{array}$

$\begin{array}{ll}\text { Index } & 213\end{array}$ 
This page is intentionally left blank 


\section{INTRODUCTION}

The theory of deterministic chaos has enjoyed during the last three decades a rapidly increasing audience of mathematicians, physicists, engineers, biologists, economists, etc. However, this type of "chaos" can be understood only as quasi-chaos in which all states of a system can be predicted and reproduced by experiments.

Meanwhile, many experiments in natural sciences have brought about hard evidence of stochastic effects. The best known example is perhaps the Brownian motion where pollen submerged in a fluid experience collisions with the molecules of the fluid and thus exhibit random motions. Other familiar examples come from fluid or plasma dynamic turbulence, optics, motions of ions in crystals, filtering theory, the problem of optimal pricing in economics, etc. The study of stochasticity was initiated in the early years of the 1900's. Einstein [1], Smoluchowsky [2] and Langevin [3] wrote pioneering investigations. This work was later resumed and extended by Ornstein and Uhlenbeck [4]. But investigation of stochastic effects in natural science became more popular only in the last three decades. Meanwhile studies are undertaken to calculate or at least approximate the effect of stochastic forces on otherwise deterministic oscillators, to investigate the stability or the transition to stochastic chaos of the latter oscillator.

To motivate the following considerations of stochastic differential equations (SDE) we introduce a few examples from natural sciences.

(a) Pendulum with Stochastic Excitations

We study the linearized pendulum motion $x(t)$ subjected to a stochastic effect, called white noise

$$
\ddot{x}+x=\beta \xi_{t},
$$


where $\beta$ is an intensity constant, $t$ is the time and $\xi_{t}$ stands for the white noise, with a single frequency and constant spectrum. For $\beta=0$ we obtain the homogeneous deterministic (non-stochastic) traditional pendulum motion. We can expect that the stochastic effect disturbs this motion and destroys the periodicity of the motion in the phase space $(x, \dot{x})$. The latter has closed solutions called limit cycles. It is an interesting task to investigate whether the solutions disintegrate into scattered points (stochastic chaos). We will cover this problem later in Section 2.3 and find that the average motion (in a sense to be defined in Section 1.2 of Chapter 1) of the pendulum is determined by the deterministic limit $(\beta=0)$ of the stochastic pendulum equation.

(b) Stochastic Growth of Populations

$\mathrm{N}(t)$ is the number of the members of a population at the time $t, \alpha$ is the constant of the deterministic growth and $\beta$ is again a constant characterizing the intensity of the white noise. Thus we study the growth problem in terms of the linear scenario

$$
\frac{\mathrm{dN}}{\mathrm{d} t}=\alpha \mathrm{N}+\beta \mathrm{N} \xi_{t} \text {. }
$$

The deterministic limit $(\beta=0)$ of this equation describes the growth of a population living on an unrestricted area with unrestricted food supply. Its solution (the number of such a population) grows exponentially. The stochastic effects, or the white noise describes a stochastic varying food supply that influences the growth of the population. We will consider this problem in the Section 2.1.1 and find again that the average of the population is given by the deterministic limit.

(c) Diffraction of Optical Waves

The transfer function $\mathrm{T}(\omega) ; \omega=\left(\omega_{1}, \omega_{2}\right)$ of a two-dimensional optical device is defined by

$$
\begin{aligned}
\mathrm{T}(\omega) & =\int_{-\infty}^{\infty} \mathrm{d} x \int_{-\infty}^{\infty} \mathrm{d} y \mathrm{~F}(x, y) \mathrm{F}^{*}\left(x-\omega_{1}, y-\omega_{2}\right) / \mathrm{N} \\
\mathrm{N} & =\int_{-\infty}^{\infty} \mathrm{d} x \int_{-\infty}^{\infty} \mathrm{d} y|\mathrm{~F}(x, y)|^{2}
\end{aligned}
$$


where $\mathrm{F}$ is a complex wave amplitude and $\mathrm{F}^{*}=\mathrm{cc}(\mathrm{F})$ is its complex conjugate. The parameter $\mathrm{N}$ denotes the normalization of $|\mathrm{F}(x, y)|^{2}$ and the variables $x$ and $y$ stand for the coordinates of the image plane. In a simplified treatment, we assume that the wave form is given by

$$
\mathrm{F}=|\mathrm{F}| \exp (-i k \Delta) ; \quad|\mathrm{F}|, k=\mathrm{const},
$$

where $k$ and $\Delta$ stand for the wave number and the phase of the waves, respectively. We suppose that the wave emerging from the optical instrument (e.g. a lens) exhibits a phase with two different deviations from a spherical structure $\Delta=\Delta_{\mathrm{c}}+\Delta_{\mathrm{r}}$ with a controlled or deterministic phase $\Delta_{c}(x, y)$ and a random phase $\Delta_{\mathrm{r}}(x, y)$ that arises from polishing the optical device or from atmospheric influences. Thus, we obtain

$$
\mathrm{T}(\omega)=\frac{1}{\mathrm{~K}} \int_{-\infty}^{\infty} \mathrm{d} x \int_{-\infty}^{\infty} \mathrm{d} y \exp \left\{i k\left[\Delta\left(x-\omega_{1}, y-\omega_{2}\right)-\Delta(x, y)\right]\right\},
$$

where $\mathrm{K}$ is used to include the normalization. In simple applications we can model the random phase using white noise with a Gaussian probability density. To evaluate the average of the transfer function $\langle\mathrm{T}(\omega)\rangle$ we need to calculate the quantity

$$
\left\langle\exp \left\{i k\left[\Delta_{\mathrm{r}}\left(x-\omega_{1}, y-\omega_{2}\right)-\Delta_{\mathrm{r}}(x, y)\right]\right\}\right\rangle .
$$

We will study the Gaussian probability density and complete the task to determine the average written in the last line in Section 1.3 of Chapter 1. An introduction to random effects in optics can be found in O'Neill [5].

\section{(d) Filtering Problems}

Suppose that we have performed experiments of a stochastic problem such as the one in (a) in an interval $t \in[0, u]$ and we obtain as result say $\mathrm{A}(v), v=[0, u]$. To improve the knowledge about the solution we repeat the experiments for $t \in[u, \mathrm{~T}]$ and we obtain $\mathrm{A}(t), t=[u, \mathrm{~T}]$. Yet due to inevitable experimental errors we do not obtain $\mathrm{A}(t)$ but a result that includes an error $\mathrm{A}(t)+$ 'noise'. The question is now how can we filter the noise away? A filter is thus, an instrument to 
clean a result and remove the noise that arises during the observation. A typical problem is where a signal with unknown frequency is transmitted (e.g. by an electronic device) and it suffers during the transmission the addition of a noise. If the transmitted signal is stochastic itself (as in the case of music) we need to develop a non-deterministic model for the signal with the aid of a stochastic differential equation. To study basic the ideas of filtering problems the reader in referred to the book of Stremler [6].

\section{(e) Fluidmechanical Turbulence}

This is the perhaps most challenging and most intricate application of statistical science. We consider here the continuum dynamics of a flow field influenced by stochastic effects. The latter arise from initial conditions (e.g. at the nozzle of a jet flow, or at the entry region of a channel flow) and/or from background noise (e.g. acoustic waves). In the simplest case, the incompressible two-dimensional flows, there are three characteristic variables (two velocity components and the pressure). These variables are governed by the Navier-Stokes equations (NSEs). The latter are a set of three nonlinear partial differential equations that included a parameter, the Reynolds number $\mathrm{R}$. The inverse of $R$ is the coefficient of the highest derivatives of the NSEs. Since turbulence occurs at intermediate to high values of the $R$, this phenomenon is the rule and not the exception in Fluid Dynamics and it occurs in parameter regions where the NSEs are singular. Nonlinear SDEs - such as the NSEs - lead additionally to the problem of the closure, where the equation governing the statistical moment of $n$th order contains moments of the $(n+1)$ th order.

Hopf [7] was the first to try to find a theoretical approach to solve the problem for the idealized case of isotropic homogenous turbulence, a flow configuration that can be approximately realized in grid flows. Hopf assumed that the turbulence is Gaussian, an assumption that facilitates the calculation of higher statistical moments of the distribution (see Section 1.3 in Chapter 1). However, later measurements showed that the assumption of a Gaussian distribution was rather unrealistic. Kraichnan [8] studied the problem again in 
the 60 's and 70's with the direct triad interaction theory in the idealized configuration of homogeneous isotropic turbulence. However, this rather involved analysis could only be applied to calculate the spectrum of very small eddies where the viscosity dominates the flow. Somewhat more progress has been achieved by the investigation of Rudenko and Chirin [9]. The latter predicted with aid of stochastic initial conditions with random phases a broad banded spectra of a nonlinear model equation. During the last two decades there was the intensive work done to investigate the Burgers equation and this research is summarized in part by Wojczinsky [10]. The Burgers equation is supposed to be a reasonable one-dimensional model of the NSEs. We will give a short account on the work done in [9] in Chapter 4. 
This page is intentionally left blank 


\section{GLOSSARY}

$\begin{array}{ll}\mathrm{AC} & \begin{array}{l}\text { almost certainly } \\ \mathrm{BC}\end{array} \\ \mathrm{dB}_{t}=\mathrm{dW}_{t}=\xi_{t} \mathrm{~d} t & \begin{array}{l}\text { boundary condition } \\ \text { differential of the Brownian motion } \\ \text { (or equivalently Wiener process) }\end{array} \\ \mathrm{cc}(a)=a^{*} & \begin{array}{l}\text { complex conjugate of } a \\ \mathrm{D}\end{array} \\ \mathrm{DF} & \text { dimension or dimensional } \\ \mathrm{DOF} & \text { degrees of freedom } \\ \delta_{i j} & \text { Kronecker delta function } \\ \delta(x) & \text { Dirac delta function } \\ \mathrm{EX} & \text { exercise at the end of a chapter } \\ \mathrm{FPE} & \text { Fokker-Planck equation } \\ \Gamma(x) & \text { gamma function } \\ \mathrm{GD} & \text { Gaussian distribution } \\ \mathrm{GPD} & \text { Gaussian probability distribution } \\ \mathrm{HPP} & \text { homogeneous Poisson process } \\ \mathrm{H} & \text { Hermite polynomial of order } n \\ \mathrm{IC} & \text { initial condition } \\ \mathrm{IID} & \text { identically independently distributed }\end{array}$


IFF if and only if

IMSL international mathematical science library

$\mathcal{L} \quad$ Laplace transform

M master, as in master equation

हैं

NSE Navier-Stokes equation

NIGD normal inverted GD

$\mathrm{N}(\mu, \sigma)$ normal distribution with $\mu$ as mean and $\sigma$ as variance

o Stratonovich theory

ODE ordinary differential equation

PD probability distribution

PDE partial differential equation

PDF probability distribution function

PSDE partial SDE

r Reynolds number

$\mathrm{RE} \quad$ random experiment

$\mathrm{RN}$ random number

RV random variable

$\operatorname{Re}(a) \quad$ real part of a complex number

$\mathrm{R}, \mathrm{C} \quad$ sets of real and complex numbers, respectively

S Prandt number

$\mathrm{SF} \quad$ stochastic function

SI stochastic integral

SDE stochastic differential equation

SLNN strong law of large numbers 


$\begin{array}{ll}\text { TPT } & \text { transition probability per unit time } \\ \text { WP } & \text { Wiener process } \\ \text { WS } & \text { Wiener sheet } \\ \text { WKB } & \text { Wentzel, Kramers, Brillouin } \\ \text { WRT } & \text { with respect to } \\ \text { W(t) } & \text { Wiener white (single frequency) noise } \\ \langle a\rangle & \text { average of a stochastic variable } a \\ \sigma^{2}=\left\langle a^{2}\right\rangle-\langle a\rangle\langle a\rangle & \text { variance } \\ \langle x \mid y\rangle,\langle x, u \mid y, v\rangle & \text { conditional averages } \\ s \wedge t & \text { minimum of } s \text { and } t \\ \forall & \text { for all values of } \\ \epsilon & \text { element of } \\ \int f(x) \mathrm{d} x & \text { short hand for } \int_{-\infty}^{\infty} f(x) \mathrm{d} x \\ \boldsymbol{\&} & \text { end of an example } \\ \square & \text { end of definition } \\ \& & \text { end of theorem }\end{array}$

